\title{
FLOWER CONSTELLATIONS FOR TELEMEDICINE SERVICES
}

\author{
M. De Sanctis ${ }^{1}$, T. Rossi ${ }^{1}$, M. Lucente ${ }^{1}$, M. Ruggieri ${ }^{\text {I }}$, C. Bruccoleri ${ }^{2}$, D. \\ Mortari $^{2}$, D. Izzo \\ ${ }^{1}$ University of Rome "Tor Vergata", Dept. of Electronic Engineering \\ Via del Politecnico 1, 00133 Rome-Italy \\ Tel. +39 067259 7258; Fax. +39 067259 7455, e-mail: ruggieri@uniroma2.it \\ 2 Texas A\&M University. \\ ${ }^{3}$ European Space Agency (ESA).
}

\begin{abstract}
Flower constellations are a particular set of satellite constellations where every satellite covers the same repeating space track. When the flower constellations are visualized on an Earth centred earth fixed reference frame, the relative orbits shows flower-shaped figures centered on the Earth. In this paper the shape and the position of a particular flower constellations has been designed for the provision of telemedicine services. Once that performance metrics of the constellation have been defined and the service targets have been identified, the performance of the flower constellation have been compared with the well known polar and Walker constellations. The particular properties of the flower constellations allow an optimized coverage of a list of targets. It was found that the flower constellations provide better performance in terms access availability and mean access time.
\end{abstract}

\section{INTRODUCTION}

The Flower Constellations (FCs) constitute an infinite set of satellite constellations characterized by axial-symmetric dynamics. They have been discovered $[1,2]$ on the way to the generalization of the concept of some existing satellite constellations. The dynamics of a FC identify a set of implicit rotating reference frames on which the satellites follow the same closed-loop relative trajectory. In particular, when the constellation axis of symmetry is chosen to be the planet's spin axis, then one of the implicit rotating reference frames coincides with a planet fixed reference frame, and, as a result, all the satellites will follow the same relative trajectory (repeating space track or compatible orbits). These relative trajectories constitute a continuous, closed-loop, symmetric pattern of flower petals.

FCs are potentially suitable for deep space observation systems, for global/regional navigation, for distributed space systems (interferometry, single and multiple-point observing systems), for telecommunications, as well as for other applications. FCs have been designed for Earth's global 
navigation, giving much better navigation performance (in terms of GDOP, ADOP, and coverage) than the existing Global Positioning System (GPS), Global Navigation Satellite System (GLONASS), and GALILEO constellations, using the same number of spacecraft or achieving the same performance using fewer satellites $[3,4]$.

In this paper, we deal with telemedicine applications which are a particular type of telecommunication service which can exploit real-time and/or storeand-forward applications.

For this type of service we can identify a specific number of locations involved in providing and accessing to the service. There are several telemedicine providers which exploit existing satellite systems for the provision of this telecommunication service. Most of the sites interested in accessing telemedicine services are located in rural areas and, hence, satellite systems are the most suitable choice for the platform service.

However, none of these satellite systems are specifically designed for telemedicine services. Furthermore they do not provide a direct connection between the service suppliers and the service customers.

In this work we design a specific $\mathrm{FC}$ for the provision of telemedicine services with the following features:

- near continuous coverage of a list of targets interested in providing and accessing the service;

- direct connection of service suppliers and service customers via satellites and Inter Satellite Links (ISLs);

- $\quad$ maximum Round Trip Time of 200 ms.

This paper is organised as follows. Section 2 introduces telemedicine services and propose a list of service targets. Section 3 discusses the theory and the design of FCs and Walker constellations; the design of the two constellations is optimised for the coverage of the targets identified in Section 2. Performance comparison of the proposed constellations is shown in Section 4, while conclusions are drawn in Section 5.

\section{TELEMEDICINE SERVICE}

In this work, the design of a FC has been applied to a particular application of telecommunication: telemedicine. Telemedicine services enable the communication and sharing of medical information in electronic form, and thus facilitate access to remote expertise. This type of service is important for large and scarcely populated countries where there is a lack of health care facilities. Immobile patients should not be required to travel long distances to receive diagnosis and medical assistance.

The objective of this work is to build up a satellite constellation able to provide medical consultancies from advanced hospitals located in Europe or USA (service suppliers) to rural and/or remote areas in Africa or Asia (service 
customers) via a pure satellite communication network; hereafter service suppliers and customers will be referred as "targets" for the satellite constellations.

The identification of the service customer targets on Earth has been performed with respect to high demographic density and lack of medical infrastructures and terrestrial communications networks. Most of the service customers are located in rural areas while service suppliers are located in metropolitan areas. In Table 1 all the identified targets are listed. We should highlight that such targets are only a sample of possible locations that could be interested to such service.

We assume that the service customers are provided with fixed or portable terminals that are used by medical/emergency teams wanting to connect to the service suppliers in order to request any of the several services that are listed in the following:

- qualified medical assistance by using teleconference or instant messaging;

- analysis of clinical data (electrocardiogram, radiological data, etc.);

- monitoring of vital parameters (blood pressure, pulse, oximetry, respiration, etc.);

- access to medical information from digital libraries;

- follow a continuing education course (tele-medicine learning).

In order to allow such services being provided, the satellite constellation must be designed for real-time applications and store-and-forward applications.

Table 1: Telemedicine service Earth targets

\begin{tabular}{|c|c|c|}
\hline Service Suppliers & Latitude & Longitude \\
\hline Fucino & 42.80 & 13.13 \\
\hline New York & 40.71 & -74.00 \\
\hline Houston & 29.76 & -95.30 \\
\hline Seattle & 47.60 & -122.33 \\
\hline Los Angeles & 34.05 & -118.24 \\
\hline
\end{tabular}

\begin{tabular}{|c|c|c|}
\hline Service Customer & Latitude & Longitude \\
\hline Lanzhou (China) & 35.96 & 104.89 \\
\hline Lusambo (Congo) & -5.69 & 23.73 \\
\hline Baliuag (Philippines) & 14.62 & 120.97 \\
\hline Mekar (Indonesia) & -6.18 & 106.63 \\
\hline Beroga (Malaysia) & 3.16 & 101.71 \\
\hline Musawa (Nigeria) & 12.00 & 8.31 \\
\hline Youngsfield (South Africa) & -33.93 & 18.46 \\
\hline
\end{tabular}


After the identification of several service suppliers and service customers, the satellite constellations will be designed with the objective to meet the following requirements:

- Near-continuous coverage of all the service customers.

- Interconnection via Inter Satellite Links (ISLs) of all the service customers and at least one service suppliers.

- $\quad$ Round Trip Time (RTT) less than $200 \mathrm{~ms}$ in order to allow interactive applications.

- Total number of satellites lower than 10 , in order to keep system cost low.

\section{CONSTELLATION DESIGN}

\section{A. Flower Constellation Design}

The FC design methodology has been extensively described in [1-5]; a summary of the main underlying principles is briefly presented in this Section for the benefit of the reader.

The name Flower Constellations has been chosen because of the compatible orbit relative trajectories in the Earth-Centred Earth-Fixed (ECEF) reference frame resemble flower petals.

A FC is a set of spacecrafts characterized by the same repeating space track, a property obtained through a suitable phasing scheme. In general FCs are characterized by 6 integer parameters:

- $\quad N_{p}$ : number of petals;

- $N_{d}:$ number of days to repeat the space track;

- $N_{s}$ : number of satellites;

- $F_{n}$ : phase numerator;

- $F_{d}$ : phase denominator;

- $F_{h}$ : phase step;

of which the first two define the semimajor axis (or the period), whereas the latter three define the satellite distribution along the relative path. Four more orbital parameters, eccentricity, inclination, argument of perigee, and Right Ascension of Ascending Node (RAAN) of the first orbit $\left(e, i, \omega, \Omega_{0}\right.$, respectively), define the orbit shape and orientation. The number of orbits is determined by the $N_{p}$ parameter and all the orbits have identical shape, inclination, and argument of perigee. They are only rotated in RAAN to obtain an even distribution about the central body.

The choice of a suitable phasing scheme is critical to reveal the most interesting dynamics obtainable by FCs. The chosen phasing scheme is a function of $\Omega_{k}$, RAAN of $\mathrm{k}^{\text {th }}$ orbit, $M_{k}\left(t_{0}\right)$ the mean anomaly at the initial time and the phasing parameters $F_{n}, F_{d}$, and $F_{h}$. The chosen scheme is designed to 
guarantee that every satellite is placed in a position compatible with the repeating space track constraint.

The FC approach provides great flexibility and interesting dynamics that reveal the presence of the so called secondary path, a relative motion of the satellites resulting in intriguing motion patterns that can be exploited to obtain useful properties as those described in [2, 3, and 5]. More details and insights and interesting properties are provided in Refs. [6,7].

Once that a list of service suppliers and service customers has been defined, by using an optimization process based on a Genetic Algorithm (GA) a FC has been designed.

The problem has been decomposed in two steps: the first is finding an orbit with a ground track that allow the observation of all the sites, the second is to find a distribution of satellites along the track that provides a good time access to the ground targets. The first step has been approached using a GA for a single satellite track, whereas for the second FCs have been utilized to extend the solution achieved in the first step to a FC of 8 satellites.

Since the GA methodology is well known and has been extensively studied, its theory will not be reviewed here; the interested reader can found ample documentation in $[8,9,10]$.

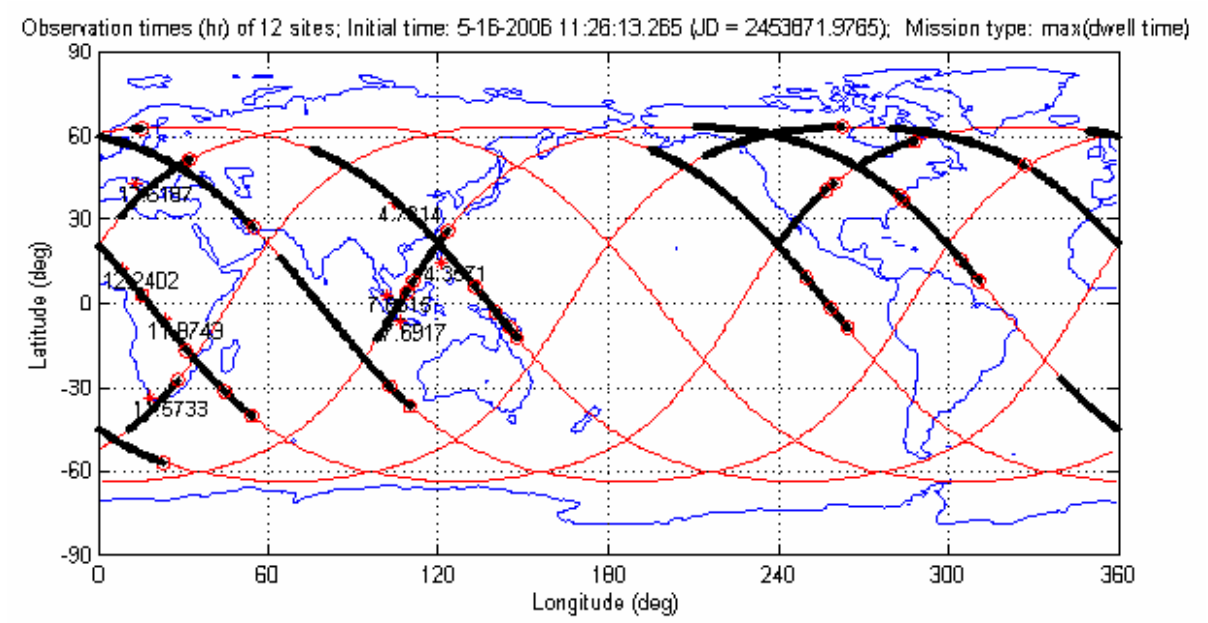

Figure 1: Repeating ground track of the FC for telemedicine.

The cost function utilized to guide the GA optimization process is designed to maximize the dwell time over each target. Since the computational load to evaluate the correct dwell time is too demanding, the dwell time is here maximized by minimizing the satellite ground relative velocity. In order to provide a preference for satellites passing over the site, the relative velocity is weighted by the ratio of the angular displacement of the target site from the 
sub-satellite point direction $(\lambda)$ with respect to the antenna field of view. These considerations yield to the following penalty function:

$$
\min J_{o b s}=\sum_{k} \alpha_{k}\left(1+\frac{\lambda_{k}}{\theta_{F O V}}\right)\left|v_{r e l}\right|
$$

where $\alpha_{\mathrm{k}}$ is the relative weight of the $k$-th site with respect to the other target sites. Therefore, by keeping the ground track spacecraft velocity $\boldsymbol{v}_{\text {rel }}$ as low as possible above target site we indirectly increase the dwell time over the site itself. The angle $\vartheta_{\mathrm{FOV}}$ is the half field of view of the on board antenna, assumed to be pointed at nadir, and $\alpha_{k}$ is the weight of the $k^{\text {th }}$ site, with $k$ $=1 . . N$, with $N$ being the number of target sites.

In order to run the GA, the design space (i.e. the chromosome) must be defined. The following parameters have been encoded in the chromosome string:

$$
\begin{aligned}
& e \varepsilon(0,1), i \varepsilon[0, \pi], \omega \varepsilon[0,2 \pi], \\
& \Omega \varepsilon[0,2 \pi], t(k) \varepsilon\left[0, t_{f}\right], k=1 . . N
\end{aligned}
$$

The semi-major axis of the desired orbit is chosen by the designer. The times $t(k)$ represent the instants in time in which the $k^{\text {th }}$ site is accessed by the spacecraft and $t_{f}$ is the final time of the simulation. The extension of the design space caused by the introduction of these time array seems counterintuitive at first. It is however justified by the need of keeping the computation time manageable: while propagating the orbit and compute these access times analytically seems to be the obvious and correct solution, this latter approach requires propagation of the orbit and a search process that slows down each GA iteration considerably. The introduction of the access times array in the design space instead allows for a quick evaluation of the cost function during each GA iterations, thus allowing a bigger population and more evaluations to be completed to achieve an improved solution. The coarse solution obtained through the GA is then refined using a gradient method search.

Some of the design variable can be kept fixed as, for instance, was done with the inclination that was kept fixed at the critical value of 63.4 degrees. The critical inclination has been chosen in order to release the need to control the drift of the perigee due to the perturbations.

Once the optimization process has been completed a FC with the parameters listed in Table 2 has been created matching the FC parameters to the ground track resulted from the optimization. 
Table 2: Flower constellations parameters.

\begin{tabular}{|l|l|}
\hline Number of Petals $\left(N_{p}\right)$ & 6 \\
\hline Number of Days $\left(N_{d}\right)$ & 1 \\
\hline Number of Satellites $\left(N_{s}\right)$ & 8 \\
\hline Phase Numerator $\left(F_{n}\right)$ & 1 \\
\hline Phase Denominator $\left(F_{d}\right)$ & 8 \\
\hline Phase Step $\left(F_{h}\right)$ & 0 \\
\hline Inclination $(i)$ & $63.4^{\circ}$ \\
\hline Perigee Height $\left(h_{p}\right)$ & $3000 \mathrm{~km}$ \\
\hline Argument of Perigee $(\omega)$ & $0^{0}$ \\
\hline RAAN & $243.77^{\circ}$ \\
\hline$M_{0}$ & $168^{\circ}$ \\
\hline
\end{tabular}

The repeating ground track of this FC is shown in Figure 1, while the relative orbit in a ECEF (Earth Centered Earth Fixed) system is shown in Figure 2 and Figure 3.

It can be noticed that six equally-spaced petals are placed above the locations of service providers and suppliers.

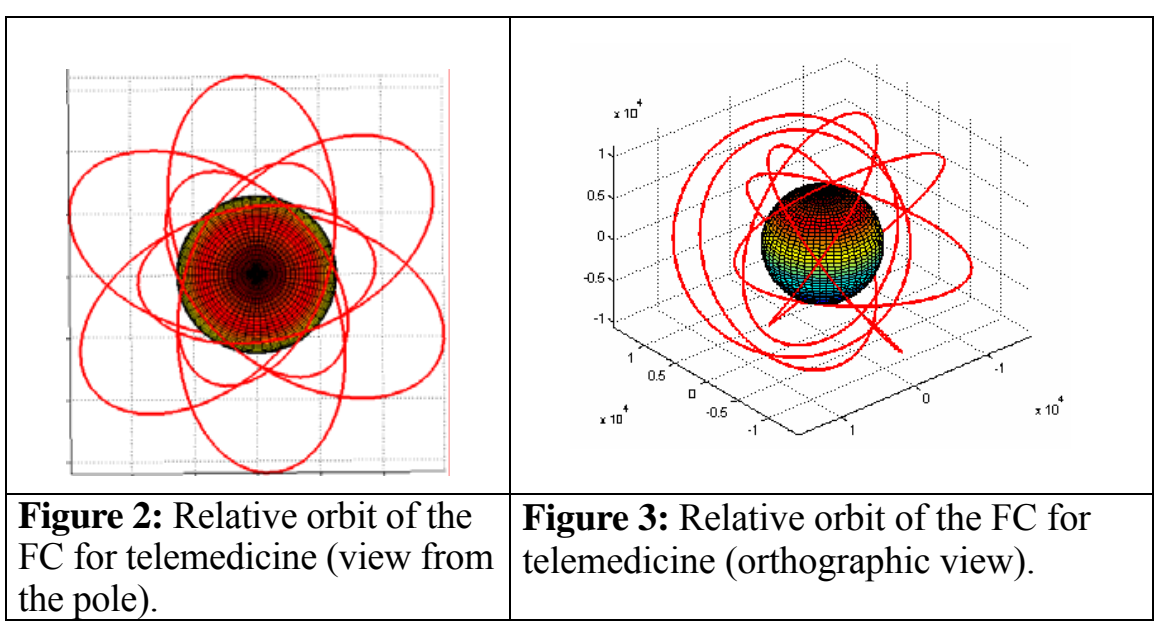

\section{B. Walker Constellation design}

Walker constellations are the classical type of satellite constellation. The design of a Walker constellation is easier with respect to the design of a Flower Constellation since the number parameters is lower. A Walker 
constellation is characterized by three integer parameters $t, p, f$ and three real parameters RAANspread, $h, i$. The parameters $t, p, f$ define respectively the number of satellites, the number of orbit planes and the relative spacing between satellites in adjacent planes. The parameters RAANspread, $h$ and $i$ define the orbit height, the inclination and the constraint on the maximum spreading of the RAAN for the satellites.

The Walker constellation is not designed to show repeating ground track. This means that the satellites belonging to a Walker constellation covers all the longitudes with the passing of time. On the other hand, the latitudinal coverage can be restricted to the equatorial regions by lowering the inclination. After the identification of the target with the highest latitude we have set the orbital inclination to 45 degrees. The number of satellites has been set to 8 as it is for the FC, while we have set the orbit altitude to 6,841 $\mathrm{km}$ in order to compare an elliptic orbit constellation (the FC) and a circular orbit constellation (the Walker costellation) with the same average satellite altitude. Furthermore, we had three choices on the number $\mathrm{p}$ of orbital planes $(8,4$ and 2$)$ and we will show the results achieved with the best configuration. Performing several evaluation of the coverage for different RAANspread, we have also found that a RAANspread $=180$ degrees is the best choice.

The parameters of the Walker constellation are listed in Table 3.

Table 3: Walker constellations parameters.

\begin{tabular}{|l|l|}
\hline Number of Satellites $(t)$ & 8 \\
\hline Number of Planes $(p)$ & 4 \\
\hline Satellites per Plane $(s=t / p)$ & 2 \\
\hline Inter Plane Spacing $(f)$ & 1 \\
\hline RAANspread & $180^{\circ}$ \\
\hline Inclination $(i)$ & $45^{\circ}$ \\
\hline Orbit Height $(h)$ & $6,841 \mathrm{~km}$ \\
\hline
\end{tabular}

\section{PERFORMANCE COMPARISON}

We are going to compare the FC and the Walker constellation in terms of the following performance metrics:

- access time (\%) between service providers and customers.

- mean access time between service providers and customers.

As previously described targets have been selected in order to model service providers and service customers.

Telecommunications chains (using only the satellite constellation network) between all service providers and customers have been created, selecting all usefull links (using 1, or none-ISL); afterwards for every chain time access 
periods have been avaluated and overlapped (in time) in order to establish the complete time-continuous coverage.

The temporal coverage, evaluated in terms of percentage of the propagation time is shown in Figure 4.

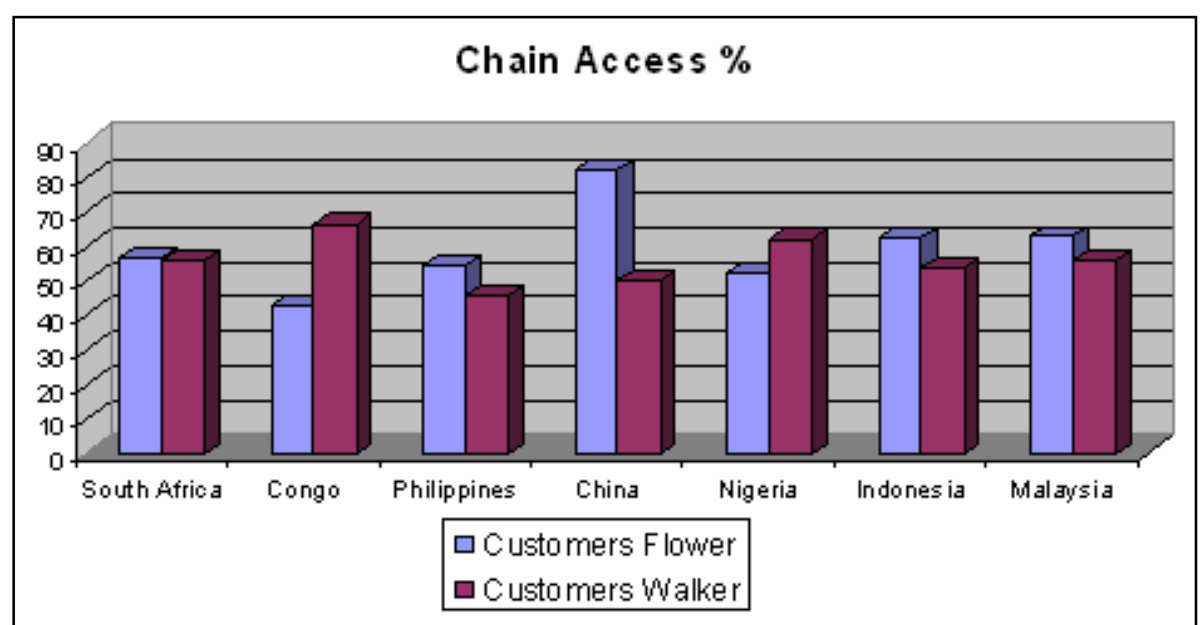

Figure 4: Chain access availability percentage performance comparison for all service customers.

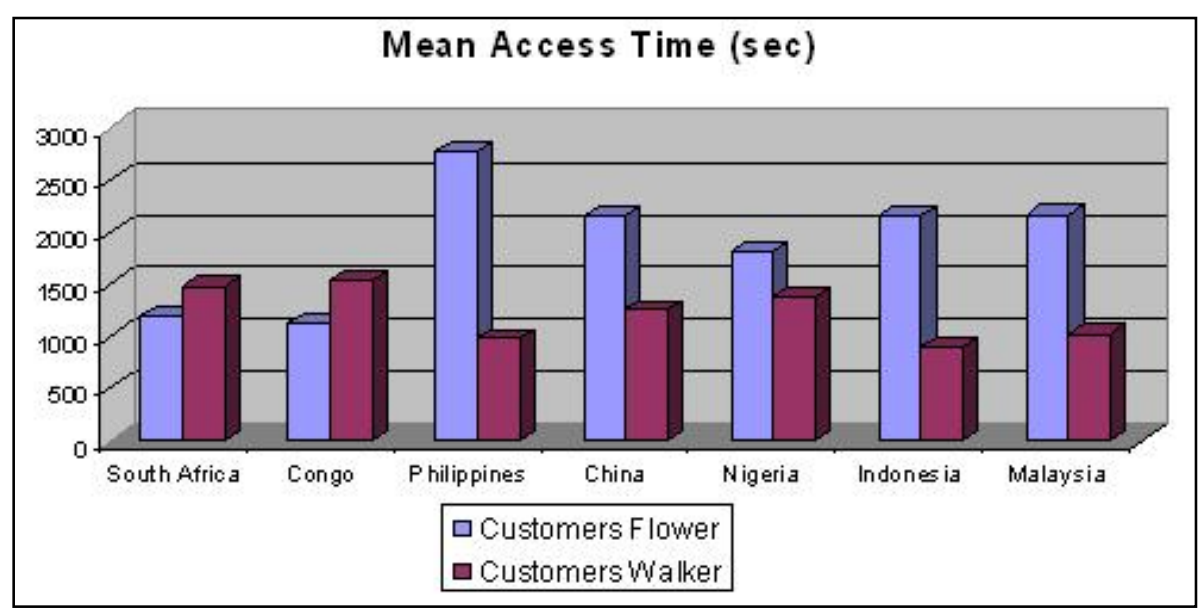

Figure 5: Mean access time performance comparison for all service customers.

The propagation time for the $\mathrm{FC}$ is set to one day $(\mathrm{Nd}=1)$ while the propagation time for the Walker constellation is set to one month.

With respect to this performance metric the bar chart in Figure 3 shows that the FC provides better performance for five customers by seven, giving an 
overall chain access of $59 \%$ versus $55 \%$ provided by the Walker constellation.

The mean chains access time which is the second performance evaluation metric is shown in Figure 5; it can be observed that, for most of the customers (five), the FC provides better results compared with the ones offered by the Walker constellation.

As a matter of fact the Walker constellation provides fractioned accesses with a mean time duration of $1220 \mathrm{sec}$. with respect to the mean time duration of $1912 \mathrm{sec}$. offered by the FC; this results means very frequent handovers for the service provided by the Walker constellation.

From this analysis it can be deduced that a FC provides better performance for telemedicine services in terms of availability and quality.

\section{CONCLUSIONS}

In this paper we designed a FC which is optimized to cover a set of targets interested in providing and accessing telemedicine services. However, the proposed optimized design can be used for a more general class of communication services and a different set of targets. It has been found that the features of the FC allow a specialised coverage of a list of targets thus providing better performance in terms of access availability with respect to a classical Walker constellation, at the expenses of a more complex design

\section{ACKNOWLEDGEMENT}

This work has been carried out in the frame of the European Space Agency Ariadna extended study: "The Flower Constellation Set and its Possible Applications".

\section{References}

[1] Mortari, D., Wilkins, M.P., and Bruccoleri, C. "The Flower Constellations," The Journal of the Astronautical Sciences, Special Issue: The John L. Junkins Astrodynamics Symposium, Vol. 52, Nos. 1 and 2, JanuaryJune 2004, pp. 107-127.

[2] Wilkins, M., Bruccoleri, C., and Mortari, D. "Constellation Design Using Flower Constellations," Paper AAS 04-208 of the 2004 Space Flight Mechanics Meeting Conference, Maui, Hawaii, February 9-13, 2004.

[3] Park, K., Wilkins, M., Bruccoleri, C., and Mortari, D. "Uniformly Distributed Flower Constellation Design Study for Global Positioning System," Paper AAS 04-297 of the 2004 Space Flight Mechanics Meeting Conference, Maui, Hawaii, February 9-13, 2004. Submitted to the ION Journal of Navigation. 
[4] Park K., Ruggieri, M., and Mortari, D. "Comparisons between GalileoSat and Global Navigation Flower Constellations," 2005 IEEE Aerospace Conference, March 5-12, 2005, Big Sky, Montana.

[5] Abdelkhalik, O. and Mortari, D. "The Two-way Orbits," 2005 IEEE Aerospace Conference, March 5-12, 2005, Big Sky, Montana.

[6] Mortari, D. and Wilkins, M.P. "The Flower Constellation Set Theory. Part I: Compatibility and Phasing," Submitted to the IEEE Transactions on Aerospace and Electronic Systems.

[7] Wilkins, M.P. and Mortari, D. "The Flower Constellation Set Theory. Part II: Secondary Paths and Equivalency," Submitted to the IEEE Transactions on Aerospace and Electronic Systems.

[8] Goldberg, D.E. "Genetic Algorithm in Search, Optimization and Machine Learning", Addison Wesley, 1989.

[9] http://en.wikipedia.org/wiki/Evolutionary_algorithm, From Wikipedia, the free encyclopedia.

[10] Wolpert, D.H., Macready, W.G. (1995), No Free Lunch Theorems for Search, Technical Report SFI-TR-95-02-010 (Santa Fe Institute). 